gr-qc/9311020, CGPG-93/11-2

\title{
Reality Conditions for Lorentzian and Euclidean Gravity in the Ashtekar Formulation
}

\author{
Guillermo A. Mena Marugán \\ Center for Gravitational Physics and Geometry, Pennsylvania State University, \\ 104 Davey Laboratory, University Park, PA 16802-6300, USA. \\ On leave from: Instituto de Matemáticas y Física Fundamental, \\ C.S.I.C., Serrano 121, 28006 Madrid, Spain.
}

November, 1993

\begin{abstract}
Using Ashtekar variables, we analyze Lorentzian and Euclidean gravity in vacuum up to a constant conformal transformation. We prove that the reality conditions are invariant under a Wick rotation of the time, and show that the compatibility of the algebra of commutators and constraints with the involution defined by the reality conditions restricts the possible values of the conformal factor to be either real or purely imaginary. In the first case, one recovers real Lorentzian general relativity. For purely imaginary conformal factors, the classical theory can be interpreted as real Euclidean gravity. The reality conditions associated with this Euclidean theory demand the hermiticity of the Ashtekar connection, but the densitized triad is represented by an anti-Hermitian operator. We also demonstrate that the Euclidean and Lorentzian sets of reality conditions lead to inequivalent quantizations of full general relativity. As a consequence, it seems impossible to obtain Lorentzian physical predictions from the quantum theory constructed with the Euclidean reality conditions.
\end{abstract}

PACS number: $04.60 .+\mathrm{n}$ 


\section{Introduction}

The Ashtekar formulation of general relativity [1-4] provides one of the most promising approaches to construct a consistent theory of quantum gravity. The Ashtekar gravitational variables, a densitized triad and a canonically conjugate complex connection [1-3], appear to be specially well-suited to deal with the type of problems that one encounters in quantizing the gravitational field. In particular, the use of connections leads in a natural way to the loop representation for quantum gravity [5-7], in which much progress has been obtained during the last five years $[7,8]$.

In the search of a quantum theory of gravity, the introduction of the Ashtekar variables has been complemented with a systematic quantization program [4] that, in addition to the non-perturbative canonical quantization scheme proposed by Dirac [9], includes the mathematical machinery needed to determine the inner product in the space of physical states $[3,10]$. One first selects an overcomplete set of complex functions on phase space that is closed under Poisson brackets. For pure gravity, for instance, the Ashtekar variables provide a set with these properties. The selected set is promoted to an abstract $*$-algebra of elementary operators in such a way that the Poisson brackets are straightforwardly translated into commutators (up to leading order in $\hbar$ ) and the complex conjugation relations between classical variables are captured in the involution operation. The corresponding $*$-relations between elementary operators are usually called reality conditions $[3,11]$. The abstract algebra of basic operators is represented then on a chosen vector space, with the physical states annihilated by all the first-class constraints of the theory [3,9]. At this point, one should find a sufficiently large number of observables for the system (i.e., operators that commute with all the constraints), derive the $*$-relations between observables that are induced by the reality conditions, and promote these relations to adjointness requirements in the Hilbert space of physical states [4]. Mathematically, 
these adjointness conditions determine the inner product in the quantum theory if the number of observables known is large enough [10]. Physically, these requirements guarantee that the spectrum (and then every quantum measurement) of any Hermitian observable is real; where we understand that an operator is Hermitian when it coincides with its $*$-conjugate.

Notice that the reality conditions between operators that are not observables cannot be promoted to adjointness conditions, because the action of these operators is not well-defined in the space of physical states. If one were able to isolate the physical degrees of freedom of the theory, the reality conditions on the non-physical degrees could be either imposed as second-class constraints before quantization or obviated by simply quantizing the associated reduced phase space. Since all complex functions on the reduced phase space are observables, the reality conditions for the reduced theory can always be promoted to adjointness requirements. The approach adopted in the non-perturbative quantization program is nevertheless more general, in the sense that one does not assume that the reduced phase space is known to carry out the quantization. However, one must keep in mind that, at the end of the day, only reality conditions on observables will play a decisive role in determining the quantum theory.

Among the problems that one finds in implementing the non-perturbative canonical quantization program, the imposition of the reality conditions that correspond to Lorentzian gravity in the Ashtekar formulation appears as one of the main technical difficulties that has to be solved in order to complete the quantization of general relativity. We recall that the Ashtekar connection is genuinely complex, its real part being given by the connection that is compatible with the triad, and its imaginary part by the extrinsic curvature $[2,3]$. As a consequence, even if one could determine the whole space of physical states and a complete set of observables for gravity, the adjointness requirements derived from the reality conditions on the complex 
Ashtekar connection and the densitized triad might be very difficult to compute and implement. The problems with reality conditions are also transmitted to the loop representation, where the $*$-relations for the elementary operators $[6,7]$, constructed using Ashtekar variables, are not even explicitly known.

Opposite to the situation in Lorentzian gravity, it has been pointed out that the reality conditions for the Ashtekar variables should be very simple in Euclidean general relativity [3]. The basic remark is that, under a Wick rotation of the time $[12,13]$, the Lorentzian extrinsic curvature transforms into $i$ times its Euclidean counterpart, the latter being real for Euclidean spaces. Therefore, the Ashtekar connection turns out to be real for Euclidean gravity, and one expects it to be represented by a Hermitian operator. The reality conditions for the densitized triad, on the other hand, should guarantee that the classical 3-metric is real. Given the apparent simplicity of these requirements, it seems natural to ask which are exactly the reality conditions for Euclidean gravity in the Ashtekar formulation, whether it is possible to adopt such conditions to quantize general relativity and if one can extract Lorentzian physical predictions from the quantum theory so obtained. The aim of this paper is to investigate the answers to these questions, and clarify the sense in which one can refer to Lorentzian and Euclidean gravity as two quantum theories built out of different sets of reality conditions.

In Sec. II, we argue that the reality conditions for the Euclideanized theory of general relativity obtained by a Wick rotation of the time coincide with those corresponding to Lorentzian gravity, so that both classical theories lead in fact to the same quantization. In Sec. III, we analyze a family of classical theories that describe either Lorentzian or Euclidean gravity up to a constant conformal transformation. We prove that, if one requires that the real densitized triad is Hermitian, the consistency of the *-operation with the algebra of elementary operators and constraints restricts the complex conformal factor to be either real or purely imaginary. 
The classical theories associated with these two different values of the conformal factor can be interpreted as real Lorentzian and real Euclidean general relativity, respectively. Sec. IV deals with the Euclidean set of reality conditions. We show there that, under very general assumptions, the quantum theories selected by the Lorentzian and Euclidean sets of reality conditions result in being inequivalent. Finally, we summarize the results in Sec.V.

\section{Lorentzian Gravity and Wick Rotation}

The Ashtekar gravitational variables can be taken as a densitized triad, $\tilde{E}_{i}^{a}$, and a $\mathrm{SO}(3)$ connection, $A_{a}^{i}$, both of them defined over a 3-manifold $\Sigma[3]$. In the following, the spatial and $\mathrm{SO}(3)$ indices will be denoted by lower case Latin letters from the beginning and the middle of the alphabet, respectively. The Ashtekar connection is canonically conjugate to the densitized triad:

$$
\left\{A_{a}^{i}(x), \tilde{E}_{j}^{b}(y)\right\}=i \delta_{a}^{b} \delta_{j}^{i} \delta^{3}(x, y),
$$

the rest of Poisson brackets between the Ashtekar variables being equal to zero. In the sector of non-degenerate metrics, the Ashtekar variables can be written in terms of the extrinsic curvature, $K_{a b}$, and the triad, $e_{i}^{a}[1-3]$,

$$
\tilde{E}_{i}^{a}=e_{i}^{a} q(e), \quad A_{a}^{i}=\Gamma_{a}^{i}(e)-i K_{a b} e^{b i},
$$

where the $\mathrm{SO}(3)$ indices are raised and lowered with the metric $\eta^{i j}=(1,1,1)$, $q^{a b}=e_{i}^{a} e^{b j}$ is the inverse 3-metric, $q(e)=\left(\operatorname{det} q_{a b}\right)^{1 / 2}$, and $\Gamma_{a}^{i}(e)$ is the $\mathrm{SO}(3)$ connection compatible with the triad:

$$
\Gamma_{a}^{i}=\frac{1}{2} \epsilon^{i j k} \underset{\sim}{E_{j b}}\left(-\partial_{a} \tilde{E}_{k}^{b}+\Gamma_{c a}^{b} \tilde{E}_{k}^{c}\right) .
$$

Here, $\epsilon^{i j k}$ is the anti-symmetric symbol, $\underset{\sim}{E_{i a}}$ denotes the inverse of the densitized triad, and $\Gamma_{b c}^{a}$ are the Christoffel symbols [14]:

$$
\Gamma_{b c}^{a}=\frac{1}{2} q^{a d}\left(\partial_{c} q_{d b}+\partial_{b} q_{d c}-\partial_{d} q_{b c}\right)
$$


Finally, the extrinsic curvature can be expressed in terms of the lapse function, $N$, the shift functions, $N^{a}$, and the time derivatives of the 3-metric [14]

$$
K_{a b}=\frac{1}{2 N}\left(\partial_{t} q_{a b}-N_{(a ; b)}\right)
$$

with $N_{(a ; b)}$ the symmetrized covariant derivative (determined by the triad) of $N_{a}=q_{a b} N^{b}$

In the Ashtekar formulation, the constraints for pure gravity adopt the simple expressions

$$
\begin{gathered}
\mathcal{G}_{i} \equiv \mathcal{D}_{a} \tilde{E}_{i}^{a}=\partial_{a} \tilde{E}_{i}^{a}+\epsilon_{i j}{ }^{k} A_{a}^{j} \tilde{E}_{k}^{a}=0, \\
\mathcal{V}_{a} \equiv \tilde{E}_{i}^{b} F_{b a}{ }^{i}=0 \\
\mathcal{S} \equiv \epsilon_{k}^{i j} \tilde{E}_{i}^{a} \tilde{E}_{j}^{b} F_{a b}{ }^{k}=0 .
\end{gathered}
$$

$F_{a b}{ }^{i}$ is the curvature of the $\mathrm{SO}(3)$ connection $A_{a}^{i}$ :

$$
F_{a b}^{i}=\partial_{a} A_{b}^{i}-\partial_{b} A_{a}^{i}+\epsilon_{j k}^{i} A_{a}^{j} A_{b}^{k}
$$

Constraints (6-8) are usually referred to as the Gauss law, the vector constraint and the scalar constraint, respectively.

It is well known that, for Lorentzian gravity, the reality conditions on the Ashtekar elementary operators can be written as [3]

$$
\left(\hat{\tilde{E}}_{i}^{a}\right)^{*}=\hat{\tilde{E}}_{i}^{a}, \quad\left(\hat{A}_{a}^{i}\right)^{*}=-\hat{A}_{a}^{i}+2 \Gamma_{a}^{i}(\hat{\tilde{E}}) .
$$

These reality conditions are the straightforward translation to the algebra of operators of the requirements that the classical densitized triad must be real (so that the metric $\tilde{\tilde{q}}^{a b}=\tilde{E}_{i}^{a} \tilde{E}^{b i}$ is positive) and the real part of the classical Ashtekar connection be given by the $\mathrm{SO}(3)$ connection compatible with the triad, which implies in turn that the extrinsic curvature $K_{a b}$ in Eq. (2) has to be real. The second of reality conditions (10) is highly non-polynomial in the densitized triad. Although it is possible to recast these reality conditions in a polynomial form [11], we will 
use in the following expressions (10) as the Lorentzian reality conditions to simplify our calculations. On the other hand, if one insists that the connection $\Gamma_{a}^{i}$ must be well-defined in terms of the densitized triad, one can always restrict his attention to the sector of non-degenerate metrics in a consistent way.

The fact that the reality conditions for Lorentzian gravity can be obtained as a direct translation of the complex conjugation relations between classical variables may lead us to misunderstand what reality conditions should be in more general cases. We recall that reality conditions are simply given by an involution in the algebra of elementary operators. When promoted to adjointness requirements, these conditions guarantee that the spectra of certain operators are real, and it is in this sense that they are related to complex conjugation conditions. This implies by no means that the $*$-relations in the algebra of operators should be exactly the operator version of the classical complex conjugation relations.

To explore this subject in more detail, let us consider the Euclideanized version of Lorentzian general relativity obtained by a Wick rotation of the time, rotation that can be accomplished by the substitution $N \rightarrow-i N$, with $N$ a real lapse function. This Euclidean theory is usually called Euclidean gravity in the literature $[12,13,15]$. However, we will keep the terminology "Euclideanized Lorentzian gravity" to refer to it; the reasons to adopt this name will become obvious in a moment. Extrapolating the results of Euclidean field theory [16], it has been frequently assumed that the quantum theory of Lorentzian general relativity can be reconstructed from the quantization of its Euclideanized version $[12,13]$. Therefore, one would expect those two quantum theories to be somehow equivalent. Actually, under a Wick rotation of the time $(N \rightarrow-i N)$, the basic Poisson brackets (1) and the gravitational constraints (6-8) remain invariant. The only important change is that the Lorentzian extrinsic curvature transforms into $i$ times its Euclidean counterpart, that is real in 
the Euclidean regime:

$$
\left(K_{E}\right)_{a b}=\frac{1}{2 N}\left(\partial_{\tau} q_{a b}-N_{(a ; b)}\right)
$$

where $\tau$ is the Euclidean time. Thus, the Euclidean Ashtekar connection takes the manifestly real expression

$$
\left(A_{E}\right)_{a}^{i}=\Gamma_{a}^{i}(e)+\left(K_{E}\right)_{a b} e^{b i}
$$

At this point, one is tempted to assert that the reality conditions for Euclideanized Lorentzian gravity are simply that the operators $\hat{\tilde{E}}_{i}^{a}$ and $\hat{A}_{a}^{i}$ must be Hermitian. However, these conditions are inconsistent with the algebra of commutators derived from Eq. (1),

$$
\left[\hat{A}_{a}^{i}(x), \hat{\tilde{E}}^{b}{ }_{j}(y)\right]=-\hbar \delta_{a}^{b} \delta_{j}^{i} \delta^{3}(x, y)
$$

and the general properties of any involution

$$
\begin{gathered}
(\lambda \hat{X})^{*}=\bar{\lambda} \hat{X}^{*} \\
(\hat{X} \hat{Y})^{*}=\hat{Y}^{*} \hat{X}^{*}
\end{gathered}
$$

where $\lambda$ is a complex number, $\hat{X}$ and $\hat{Y}$ are two generic operators, and ${ }^{--}$denotes complex conjugation. Note that, in particular, Eq. (15) implies that $\hat{1}^{*}=\hat{1}$.

To prove such an inconsistency, it is enough to take the $*$-conjugate of the commutator (13) and use Eqs. (14,15). If both the densitized triad and the Ashtekar connection were Hermitian, one would arrive at the conclusion that their commutator should be given by the right-hand side of Eq. (13) but with a flip of sign, in clear contradiction with our original assumptions.

In fact, it is a standard result of Euclidean field theory that the *-relations for the Euclidean fields are given by the composition of the time reversal and the complex conjugation operations [16]. Choosing the manifold $\Sigma$ as the zero time section of the four-dimensional space, and taking into account that the densitized triad remains invariant under time reversal, while the Euclidean extrinsic curvature changes its sign, 
we conclude that (if the results of Euclidean field theory are applicable to general relativity) the reality conditions for Euclideanized Lorentzian gravity coincide with the Lorentzian conditions (10). Therefore, the *-algebra of elementary operators and constraints for Lorentzian general relativity and its Euclideanized version are exactly the same, and both classical theories lead indeed to the same quantization.

It may seem strange that a quantum theory can describe simultaneously two classical theories whose associated equations of motion are of Lorentzian and Euclidean type, respectively. We notice, nevertheless, that both kinds of dynamics are related by an analytic continuation of the time coordinate. From the point of view of the algebraic structures and constraints that determine the quantum theory there is however no concept of time a priori. It is only after introducing a time parameter in the quantum theory (an intrinsic time) that one can recover the notion of dynamical evolution and, presumably, the classical equations of motion in a certain limit. As it usually happens in the WKB approximation employed in quantum cosmology [17-19], we expect then that, for real values of the introduced time parameter, one can regain Lorentzian dynamics, while, for imaginary times obtained by means of an analytic continuation, the dynamical regime should become Euclidean.

\section{Conformally Lorentzian and Euclidean Gravity}

We are interested in determining whether it is possible to find a set of reality conditions, other than those given by Eq. (10), that correspond in some sense to a classical Euclidean theory of gravity and such that, in particular, the Ashtekar connection is represented by a Hermitian operator. To explore this topic, we will analyze in this section a family of classical theories that, at least in vacuum, lead to either Lorentzian or Euclidean dynamics.

We will consider those sections of the complex phase space of general relativity for which the 4-metric is either Lorentzian or Euclidean, modulo a constant conformal 
transformation:

$$
d s^{2}=\Omega^{2}\left(d s_{P}\right)^{2} .
$$

Here, $\Omega$ is a complex constant whose absolute value is equal to the unity (otherwise, $|\Omega|$ can be absorbed into the line element $\left.\left(d s_{P}\right)^{2}\right)$,

$$
\Omega=e^{i \Theta}, \quad \Theta \in[0,2 \pi) .
$$

The 3-metric $\left(q_{P}\right)_{a b}$ determined by $\left(d s_{P}\right)^{2}$ will be assumed to be real and nonnegative. The lapse and shift functions associated with $\left(d s_{P}\right)^{2}$ will be denoted, respectively, by $N_{P}$ and $N_{P}^{a}$. Although one can take $N_{P}$ and $N_{P}^{a}$ to be complex in general, we will restrict our attention to the cases $N_{P}^{a}, N_{P} \in \mathbb{R}$, corresponding to Lorentzian metrics, and $N_{P}^{a}$ real and $N_{P}$ imaginary, for which the line element $\left(d s_{P}\right)^{2}$ results in being Euclidean.

We notice that the constant conformal factor $\Omega^{2}$ will decouple from the equations of motion in pure gravity. As a consequence, the evolution of the real metric $\left(q_{P}\right)_{a b}$ will be completely consistent both in the Lorentzian and the Euclidean regimes, as it actually happens in the particular case $\Omega^{2}=1$.

It is a simple exercise to show that, under the conformal transformation defined by Eq. (16), the lapse and shift functions and the 3-metric of $d s^{2}$ and $\left(d s_{P}\right)^{2}$ are related by

$$
N=\Omega N_{P}, \quad N^{a}=N_{P}^{a}, \quad q_{a b}=\Omega^{2}\left(q_{P}\right)_{a b},
$$

so that the transformation law for the triad is

$$
e_{i}^{a}=\Omega^{-1}\left(e_{P}\right)_{i}^{a}
$$

Using Eqs. $(18,19)$ and Eqs. (2-5), it is easy to derive the relations between the densitized triads and the $\mathrm{SO}(3)$ connections of the two considered four-metrics:

$$
\tilde{E}_{i}^{a}=\Omega^{2}\left(\tilde{E}_{P}\right)_{i}^{a}, \quad A_{a}^{i}=\left(A_{P}\right)_{a}^{i} .
$$


Substituting these equations in the gravitational constraints (6-8), and taking into account the definition (9) of the $\mathrm{SO}(3)$ curvature $F_{a b}{ }^{i}$, we obtain that

$$
\mathcal{G}_{i}=\Omega^{2}\left(\mathcal{G}_{P}\right)_{i}=0, \quad \mathcal{V}_{a}=\Omega^{2}\left(\mathcal{V}_{P}\right)_{a}=0, \quad \mathcal{S}=\Omega^{4} \mathcal{S}_{P}=0
$$

with the subindex $P$ denoting evaluation at $\left(\tilde{E}_{P}\right)_{i}^{a}$ and $\left(A_{P}\right)_{a}^{i}$. Therefore, the gravitational constraints for the Ashtekar variables $\tilde{E}_{i}^{a}$ and $A_{a}^{i}$ can be equivalently written by simply evaluating them at the conformally transformed variables.

In the Ashtekar formulation, the classical time evolution is generated by the Hamiltonian

$$
H=\int_{\Sigma} d^{3} x\left(\frac{1}{2} N_{\sim} \mathcal{S}-i N^{a}\left(\mathcal{V}_{a}-A_{a}^{i} \mathcal{G}_{i}\right)+i N^{i} \mathcal{G}_{i}\right)+\text { Surface Terms }
$$

Here, $\mathcal{G}_{i}, \mathcal{V}_{a}$ and $\mathcal{S}$ are given by Eqs. (6-8), $\underset{\sim}{N}=N q^{-1 / 2}$ is the densitized lapse function, $N^{a}$ is the shift function and $N^{i}$ is a $\mathrm{SO}(3)$ Lagrange multiplier [3]. The surface terms in Eq. (22) are introduced to render $H$ finite, and depend on the conditions imposed on the Ashtekar variables (and the Lagrange multipliers) on the boundary of the 3 -manifold $\Sigma[2,3]$. Since these surface terms do not alter the dynamical equations of $\tilde{E}_{i}^{a}$ and $A_{a}^{i}$, we will obviate them in the discussion to follow.

The Hamiltonian (22) leads to the equations of motion $[2,3]$

$$
\begin{gathered}
\dot{\tilde{E}}_{i}^{a}=\left\{\tilde{E}_{i}^{a}, H\right\}=-i \mathcal{D}_{b}\left(\underset{\sim}{N} \epsilon_{i}{ }^{j k} \tilde{E}_{j}^{a} \tilde{E}_{k}^{b}\right)+\mathcal{L}_{\bar{N}}\left(\tilde{E}_{i}^{a}\right)+N^{k} \tilde{E}_{j}^{a} \epsilon_{i}{ }^{j}{ }_{k}, \\
\dot{A}_{a}^{i}=\left\{A_{a}^{i}, H\right\}=i \underset{\sim}{N} \epsilon_{k}^{i j} \tilde{E}_{j}^{b} F_{a b}{ }^{k}+\mathcal{L}_{\bar{N}} A_{a}^{i}+\mathcal{D}_{a} N^{i},
\end{gathered}
$$

where the dot denotes time derivative, $\mathcal{L}_{\bar{N}}$ is the Lie derivative:

$$
\mathcal{L}_{\bar{N}}\left(\tilde{E}_{i}^{a}\right)=\partial_{b}\left(N^{b} \tilde{E}_{i}^{a}\right)-\tilde{E}_{i}^{b} \partial_{b} N^{a}, \quad \mathcal{L}_{\bar{N}} A_{a}^{i}=N^{b} \partial_{b} A_{a}^{i}+A_{b}^{i} \partial_{a} N^{b},
$$

and $\mathcal{D}_{b}$ is the derivative operator defined by the Ashtekar connection:

$$
\begin{gathered}
\mathcal{D}_{b}\left(\underset{\sim}{N} \epsilon_{i}^{j k} \tilde{E}_{j}^{a} \tilde{E}_{k}^{b}\right)=\partial_{b}\left(\underset{\sim}{N} \epsilon_{i}^{j k} \tilde{E}_{j}^{a} \tilde{E}_{k}^{b}\right)+\epsilon_{i l}{ }^{m} A_{b}^{l} \underset{\sim}{N} \epsilon_{m}^{j k} \tilde{E}_{j}^{a} \tilde{E}_{k}^{b}, \\
\mathcal{D}_{a} N^{i}=\partial_{a} N^{i}+\epsilon_{j k}^{i} A_{a}^{j} N^{k} .
\end{gathered}
$$


Under the conformal transformation (16), the $\mathrm{SO}(3)$ Lagrange multiplier $N^{i}$ remains unchanged, $N^{i}=N_{p}^{i}$. From the definition of $\underset{\sim}{N}$ and Eq. (18), we also conclude that

$$
\underset{\sim}{N}=\Omega^{-2} N_{P}, \quad N^{a}=N_{P}^{a} .
$$

It is then possible to see that the dynamical equations $(23,24)$ are still valid when evaluated at the Lagrange multipliers and Ashtekar variables determined by $\left(d s_{P}\right)^{2}$. To prove this statement, it suffices to realize that Eqs. (1), (20-22) and (28), together with $N^{i}=N_{P}^{i}$, imply that

$$
\begin{gathered}
\left\{\left(A_{P}\right)_{a}^{i}(x),\left(\tilde{E}_{P}\right)_{j}^{b}(y)\right\}=\Omega^{-2} i \delta_{a}^{b} \delta_{j}^{i} \delta^{3}(x, y), \\
H=\Omega^{2} H_{P} .
\end{gathered}
$$

Thus, the Poisson brackets of $\left(\tilde{E}_{P}\right)_{i}^{a}$ and $\left(A_{P}\right)_{a}^{i}$ with $H$ get a factor $\Omega^{2}$ from the conformal transformation of the Hamiltonian, and a factor $\Omega^{-2}$ because of the modification of the basic Poisson brackets between the densitized triad and the Ashtekar connection. Therefore, the equations of motion for $\left(\tilde{E}_{P}\right)_{i}^{a}$ and $\left(A_{P}\right)_{a}^{i}$ coincide with the right-hand side of Eqs. $(23,24)$ evaluated at the variables associated with the line element $\left(d s_{P}\right)^{2}$. This result can also be obtained by substituting Eqs. (20) and (28) (and $N^{i}=N_{P}^{i}$ ) into expressions (23-27).

For $N_{P}^{i}$ and $N_{P}^{a}$ real, the time evolution defined by Eq. (23) respects the reality of the densitized triad $\left(\tilde{E}_{P}\right)_{i}^{a}$ in the Lorentzian regime $\left(N_{P} \in \mathbb{R}\right)$ provided that the real part of $\left(A_{P}\right)_{a}^{i}$ is equal to the connection $\Gamma_{a}^{i}\left(\tilde{E}_{P}\right)[2,3]$. On the other hand, it is straightforward to check that, for real $N_{P}^{i}$ and $N_{P}^{a}$, the equations of motion $(23,24)$ are also consistent with the reality of the densitized triad $\left(\tilde{E}_{P}\right)_{i}^{a}$ and the $\mathrm{SO}(3)$ connection $\left(A_{P}\right)_{a}^{i}$ in the Euclidean regime, i.e., for imaginary lapse functions $N_{P}$, for which $i N_{P} \in \mathbb{R}$. As we had anticipated, the sections of the complex phase space of general relativity with real densitized triads up to a constant complex factor can then be employed to describe, at least in vacuum, both classical Lorentzian and 
Euclidean gravity, and the specific regime considered depends on the values taken by the complexified time coordinate.

Let us try to find now the sets of reality conditions that correspond to this family of classical theories. We first note that the reality conditions for the Lorentzian and Euclidean theories analyzed here should be essentially the same for each complex conformal factor $\Omega^{2}$, because, with $\Omega^{2}$ fixed, these two types of dynamical regime are always related by an analytic continuation of the time, and then the arguments presented in the previous section for the particular case $\Omega^{2}=1$ can be applied as well to all these models.

Since the densitized triad $\left(\tilde{E}_{P}\right)_{i}^{a}$ is real in all the theories that we are studying, we will require $\left(\hat{\tilde{E}}_{P}\right)_{i}^{a}$ to be Hermitian as part of the reality conditions. Thus,

$$
\left(\hat{\tilde{E}}_{i}^{a}\right)^{*}=\bar{\Omega}^{2}\left(\left(\hat{\tilde{E}}_{P}\right)_{i}^{a}\right)^{*}=\Omega^{-2}\left(\hat{\tilde{E}}_{P}\right)_{i}^{a}=\Omega^{-4} \hat{\tilde{E}}_{i}^{a} .
$$

The reason to justify this assumption is that it ensures that any possible real observable constructed only from the metric $\left(q_{P}\right)_{a b}$ will be represented in the quantum theory by a self-adjoint operator, so that its expectation values will always be real. The admissible reality conditions for the $\mathrm{SO}(3)$ connection $A_{a}^{i}$ are then severely restricted by the compatibility of the algebra of commutators (13) with the properties of the $*$-relation $(14,15)$. Defining the triadic extrinsic curvature as

$$
-i \hat{K}_{a}^{i}=\hat{A}_{a}^{i}-\Gamma_{a}^{i}(\hat{\tilde{E}}),
$$

and taking the $*$-conjugate of Eq. (13), we arrive at the result

$$
\left(\left[i \hat{K}_{a}^{i}(x), \hat{\tilde{E}}^{b}{ }_{j}(y)\right]\right)^{*}=-\hbar \delta_{a}^{b} \delta_{j}^{i} \delta^{3}(x, y)=\Omega^{-4} i\left[\left(\hat{K}_{a}^{i}\right)^{*}(x), \hat{\tilde{E}}^{b}{ }_{j}(y)\right] .
$$

Then, the reality conditions for $\hat{K}_{a}^{i}$ must be of the form

$$
\left(\hat{K}_{a}^{i}\right)^{*}=\Omega^{4} \hat{K}_{a}^{i}+f_{a}^{i}(\hat{\tilde{E}}) .
$$

Here, $f_{a}^{i}$ is a function of the densitized triad (and its spatial derivatives) still to be determined. 
It is worth remarking that the classical analogue of $\hat{K}_{a}^{i}$ will be, in general, a complex triadic extrinsic curvature, its value depending on that of the complex lapse function $N_{P}$. It thus seems natural not to assume any given reality condition for $\hat{K}_{a}^{i}$, but to deduce it from the consistency of the algebraic structures.

For the $*$-operation to be an involution, we have to require also that

$$
\hat{K}_{a}^{i}=\left(\hat{K}_{a}^{i}\right)^{* *}=\hat{K}_{a}^{i}+\Omega^{-4} f_{a}^{i}(\hat{\tilde{E}})+\bar{f}_{a}^{i}\left(\Omega^{-4} \hat{\tilde{E}}\right),
$$

and so

$$
\bar{f}_{a}^{i}\left(\Omega^{-4} \hat{\tilde{E}}\right)=-\Omega^{-4} f_{a}^{i}(\hat{\tilde{E}})
$$

On the other hand, the reality conditions should guarantee that the $*$-conjugate of the operator constraints do not lead to any new constraint in the system different from those originally imposed. Otherwise, the consistency of the quantization procedure will demand the introduction of additional constraints that were not present in the classical theory from which one started. This statement has never appeared in the literature, although it is clear that it has always been implicitly assumed.

Let us study first the Gauss law (6), which can be equivalently written as

$$
\mathcal{G}_{i} \equiv-i \epsilon_{i j}{ }^{k} \hat{K}_{a}^{j} \hat{\tilde{E}}_{k}^{a}=0
$$

From now on, we will employ in our calculations the symmetric factor ordering for all the products of the operators $\hat{K}_{a}^{i}$ and $\hat{\tilde{E}}_{i}^{a}$, even if this ordering is not displayed explicitly. Applying the *-operation to Eq. (37), and using reality conditions (31) and (34), we conclude that, for all possible values of $\Omega, \mathcal{G}_{i}{ }^{*}=0$ is equivalent to $\mathcal{G}_{i}=0$ if and only if

$$
\epsilon_{i j}^{k} f_{a}^{j}(\hat{\tilde{E}}) \hat{\tilde{E}}_{k}^{a}=0
$$

Modulo the Gauss law and the Bianchi identities, the vector constraint (7) can be expressed as [3]

$$
\mathcal{V}_{a} \equiv-i D_{b}\left(\hat{K}_{a}^{i} \hat{\tilde{E}}_{i}^{b}-\hat{K}_{c}^{i} \hat{\tilde{E}}_{i}^{c} \delta_{a}^{b}\right)=0
$$


with $D_{b}$ the derivative operator compatible with the triad, i.e., $D_{b}\left(\hat{\tilde{E}}_{i}^{a}\right)=0$ and

$$
D_{b}\left(\hat{K}_{a}^{i}\right)=\partial_{b} \hat{K}_{a}^{i}+\epsilon_{j k}^{i} \Gamma_{b}^{j}(\hat{\tilde{E}}) \hat{K}_{a}^{k}+\Gamma_{b a}^{c}(\hat{\tilde{E}}) \hat{K}_{c}^{i}
$$

From Eqs. (31) and (34), it is then easy to check that $\mathcal{V}_{a}{ }^{*}=0$ provided that $\mathcal{V}_{a}=0$ (and vice versa) only if $f_{a}^{i}$ satisfies the requirement

$$
D_{b}\left(f_{a}^{i}(\hat{\tilde{E}}) \hat{\tilde{E}}_{i}^{b}-f_{c}^{i}(\hat{\tilde{E}}) \hat{\tilde{E}}_{i}^{c} \delta_{a}^{b}\right)=0
$$

similar to the constraint (39) on $\hat{K}_{a}^{i}$.

Finally, the scalar constraint (8) can be rewritten, modulo the Gauss law [3],

$$
\mathcal{S} \equiv q^{2}(\hat{\tilde{E}}) R(\hat{\tilde{E}})+2 \hat{K}_{a}^{i} \hat{K}_{b}^{j} \hat{\tilde{E}}_{[i}^{a} \hat{\tilde{E}}_{j]}^{b}=0
$$

where the brackets denote antisymmetrization, $R(\hat{\tilde{E}})$ is the operator associated with the scalar curvature of the 3 -metric $q_{a b}$, and $q^{2}(\hat{\tilde{E}})$ corresponds to the determinant of that metric:

$$
q^{2}(\hat{\tilde{E}})=\frac{1}{6} \epsilon^{i j k} \epsilon_{a b c} \hat{\tilde{E}}_{i}^{a} \hat{\tilde{E}}_{j}^{b} \hat{\tilde{E}}_{k}^{c}
$$

Taking the $*$-conjugate of Eq. (42), and subtracting from it the original constraint, we arrive after some trivial manipulations at the condition

$$
\left(1-\Omega^{8}\right) q^{2}(\hat{\tilde{E}}) R(\hat{\tilde{E}})+2 f_{a}^{i}(\hat{\tilde{E}}) f_{b}^{j}(\hat{\tilde{E}}) \hat{\tilde{E}}_{[i}^{a} \hat{\tilde{E}}_{j]}^{b}=-4 \Omega^{4} f_{a}^{i}(\hat{\tilde{E}}) \hat{K}_{b}^{j} \hat{\tilde{E}}_{[i}^{a} \hat{\tilde{E}}^{b}{ }_{j]},
$$

where we have employed that, under the conformal transformation $\tilde{E}_{i}^{a} \rightarrow \Omega^{-4} \tilde{E}_{i}^{a}$,

$$
R\left(\Omega^{-4} \tilde{E}\right) \equiv R\left(\Omega^{-4} q_{a b}\right)=\Omega^{4} R\left(q_{a b}\right) \equiv \Omega^{4} R(\tilde{E})
$$

Since $f_{a}^{i}$ is a function of the densitized triad and its spatial derivatives, and Eq. (44) includes also the extrinsic curvature $\hat{K}_{a}^{i}$, such equation can be satisfied without introducing a new constraint in the system only if it can be derived from the original gravitational constraints (6-8) for some particular choice of $f_{a}^{i}$. We note that expression (44) establishes a relation between an homogeneous function of 
degree one in the extrinsic curvature and another homogeneous function of degree equal to zero, both of them dependent on the densitized triad. Because of this fact, the scalar constraint (42) cannot be used to eliminate the dependence on the extrinsic curvature in Eq. (44), for it can only reduce the order of homogeneity in $\hat{K}_{a}^{i}$ by a multiple of 2 . On the other hand, the vector constraint (39) is homogeneous of degree one in the extrinsic curvature, and it can only be employed to substitute some components of the extrinsic curvature as linear combinations (depending on the triad) of other components and their spatial derivatives. Therefore, equation (44) is functionally independent of the scalar and vector constraints.

Finally, the Gauss law (37) implies that $\hat{\tilde{K}}_{j}^{i}=\hat{K}_{a}^{i} \hat{\tilde{E}}_{j}^{a}$ is symmetric in the $\mathrm{SO}(3)$ indices. Rewritten then the right-hand side of Eq. (44) as

$$
-2 \Omega^{4}\left(\tilde{f}_{i}^{i}(\hat{\tilde{E}}) \hat{\tilde{K}}_{j}^{j}-\tilde{f}_{j}^{i}(\hat{\tilde{E}}) \hat{\tilde{K}}_{i}^{j}\right)
$$

and taking into account that $\tilde{f}_{j}^{i}(\hat{\tilde{E}})=f_{a}^{i}(\hat{\tilde{E}}) \hat{\tilde{E}}_{j}^{a}$ is symmetric because of condition (38), we conclude that the sole way in which relation (44) can be satisfied, for $\hat{\tilde{K}}_{i}{ }_{i}$ a generic $\mathrm{SO}(3)$ symmetric tensor operator that is functionally independent of the densitized triad, is that both sides of the considered equation vanish. In particular, expression (46) must be identically zero, whichever the values of the symmetric extrinsic curvature may be. In order to verify this requirement, the function $\tilde{f}_{j}^{i}$ must vanish, as one can check after some trivial calculations. Then, $f_{a}^{i}(\hat{\tilde{E}})=\tilde{f}_{j}^{i}\left(\hat{\tilde{E}}^{\hat{E}_{\sim}^{j}}{ }_{a}\right.$ has to be equal to zero (at least for non-degenerate metrics; if we want to extend our conclusions to the degenerate case, we have to assume the continuity of $f_{a}^{i}$ as a function of the densitized triad).

Obviously, for $f_{a}^{i}=0$, the additional conditions (36), (38) and (41) are immediately fulfilled. The only consistency demand that we have not discussed yet is the vanishing of the left-hand side of Eq. (44) at $f_{a}^{i}=0$ :

$$
\left(1-\Omega^{8}\right) q^{2}(\hat{\tilde{E}}) R(\hat{\tilde{E}})=0 .
$$


This condition can be satisfied, for instance, by restricting our attention to flat minisuperspace models. For these models, we have proved then that (at least in vacuum) it is possible to adopt sets of reality conditions of the form $\left(\hat{\tilde{E}}_{i}^{a}\right)^{*}=\Omega^{-4} \hat{\tilde{E}}_{i}^{a}$ and $\left(\hat{A}_{a}^{i}\right)^{*}=-\Omega^{4} A_{a}^{i}$ with $\Omega$ any complex constant $(|\Omega|=1)$. In the general case, however, we will have that $q^{2}(\tilde{E}) R(\tilde{E})$ is different from zero. Thus, in the full theory of gravity one is forced to demand that $\Omega^{8}=1$. In other words, once one assumes that the densitized triad $\left(\hat{\tilde{E}}_{P}\right)_{i}^{a}$ (that is real in the classical theory) is Hermitian, the compatibility of the reality conditions with the algebraic structures and constraints of general relativity restricts the complex conformal factor to be such that $\Omega^{8}=1$. For those theories with other constant values of $\Omega$, the densitized triad $\hat{\tilde{E}}_{i}^{a}$ cannot be a Hermitian operator up to a complex constant factor, and any classical real observable that might be constructed from the 3-metric will have in general genuine complex expectation values in the corresponding quantum theory.

Let us focus then our discussion on the case $\Omega^{8}=1$. Since reality conditions (31) and (34), with $f_{a}^{i}=0$, depend just on the fourth power of $\Omega$, it will suffice to consider two possible values for the conformal factor: $\Omega^{2}=1$ and $\Omega^{2}=-i$. The case $\Omega^{2}=1$ was analyzed in Sec. 2; it corresponds to the Lorentzian sector of general relativity,

with reality conditions given by $\left(\hat{\tilde{E}}_{i}^{a}\right)^{*}=\hat{\tilde{E}}_{i}^{a}$ and $\left(\hat{A}_{a}^{i}\right)^{*}=-\hat{A}_{a}^{i}+2 \Gamma_{a}^{i}(\hat{\tilde{E}})$. On the other hand, we will argue in the next section that the reality conditions for $\Omega^{2}=-i$ can be associated in a natural way with the Euclidean sector of pure gravity.

\section{Reality Conditions for Euclidean Gravity}

When the conformal factor $\Omega^{2}$ is purely imaginary, reality conditions (31) and (34) can be rewritten

$$
\left(\hat{\tilde{E}}_{i}^{a}\right)^{*}=-\hat{\tilde{E}}_{i}^{a}, \quad\left(\hat{A}_{a}^{i}\right)^{*}=\hat{A}_{a}^{i},
$$

where we have used the definition (32) for the triadic extrinsic curvature. The second of these relations affirms that the Ashtekar connection is Hermitian, as one would 
expect it to happen in Euclidean gravity. In fact, it is not difficult to show that reality conditions (48) are simply the direct translations of the complex conjugation relations for real Euclidean gravity in the Ashtekar formalism.

To prove this statement, let us study the classical theory obtained from Lorentzian general relativity by a Wick rotation of the time and a constant and purely imaginary conformal transformation. In the Ashtekar formulation, the gravitational action can be expressed [3]

$$
S=\int d t\left[\int_{\Sigma} d^{3} x\left(-i \tilde{E}_{i}^{a} \dot{A}_{a}^{i}\right)-H^{\prime}\right]
$$

where $H^{\prime}$ coincides formally with the Hamiltonian $H$ given by Eq. (22) without surface terms. This action is equal on shell to the Hilbert-Einstein action of general relativity $[3,11]$, which is real in the Lorentzian regime. Under a Wick rotation of the time, the Lorentzian action transforms into $i$ times the action for Euclidean gravity $[12,15,18]$, the latter being real for Euclidean 4-metrics. On the other hand, the transformation laws (20) and (30) (still valid for $H^{\prime}$ ) imply that, for the theory with conformal factor $\Omega^{2}=-i$, the Ashtekar action is exactly $-i$ times the action of the non-transformed variables. Therefore, in the classical theory with imaginary conformal factor and Wick rotated time, the gravitational action (49) turns out to coincide on shell with the real classical action of pure Euclidean gravity. In terms of the Ashtekar variables, the Euclidean theory is described by the densitized triad $\left(\tilde{E}_{P}\right)_{i}^{a}=i \tilde{E}_{i}^{a}$ and the connection $\left(A_{P}\right)_{a}^{i}=A_{a}^{i}$, which, in agreement with our previous remarks, are both real in the classical theory and Hermitian when considered as operators. Thus, reality conditions (48) ensure that all real observables constructed from the Euclidean 3-metric and extrinsic curvature are self-adjoint in the quantum theory.

Another topic that we want to address is whether reality conditions (10) and (48), that correspond, respectively, to Lorentzian and Euclidean gravity, determine at the end of the day unitarily equivalent quantum theories. If the answer were in 
the positive, one could use the simple reality conditions (48) to quantize general relativity, and obtain from that quantum theory physical predictions for Lorentzian gravity [20]. However, we will prove that, under very general assumptions, this is not indeed the case.

Reality conditions (10) and (48) will lead to equivalent quantum theories if there exists an isomorphism between their respective $*$-algebras of elementary operators that leaves invariant the gravitational constraints. In the following, we will use the symbol $I$ to denote this isomorphism, and the subindex 0 to refer to those operators that belong to the Euclidean *-algebra.

The isomorphism $I$ must satisfy the requirements

$$
\begin{gathered}
I\left(\hat{X}_{0} \hat{Y}_{0}\right)=I\left(\hat{X}_{0}\right) I\left(\hat{Y}_{0}\right), \\
I\left(\hat{X}_{0}^{*}\right)=\left(I\left(\hat{X}_{0}\right)\right)^{*}, \\
I\left(\left[\hat{X}_{0}, \hat{Y}_{0}\right]\right)=\left[I\left(\hat{X}_{0}\right), I\left(\hat{Y}_{0}\right)\right],
\end{gathered}
$$

with $\hat{X}_{0}$ and $\hat{Y}_{0}$ any two operators. In particular, from the first of these equations we get that $I\left(\hat{1}_{0}\right)=\hat{1}$.

Since the operators $i\left(\hat{\tilde{E}}_{0}\right)_{i}^{a}$ and $\hat{\tilde{E}}_{i}^{a}$ are both Hermitian, and both of them describe classical real densitized triads, it seems natural to assume that

$$
I\left(\left(\hat{\tilde{E}}_{0}\right)_{i}^{a}\right)=-i \hat{\tilde{E}}_{i}^{a} \text {. }
$$

The consistency condition $I\left(\hat{\tilde{E}}_{0}{ }^{*}\right)=\left(I\left(\hat{\tilde{E}}_{0}\right)\right)^{*}$ is then automatically verified.

The isomorphism for the connection operator $\left(\hat{A}_{0}\right)_{a}^{i}$ can be derived from Eqs. (13) and $(52,53)$, for they imply that

$$
I\left(\left[\left(\hat{A}_{0}\right)_{a}^{i}(x),\left(\hat{\tilde{E}}_{0}\right)^{b}{ }_{j}(y)\right]\right)=\left[I\left(\left(\hat{A}_{0}\right)_{a}^{i}(x)\right),-i \hat{\tilde{E}}^{b}{ }_{j}(y)\right]=-\hbar \delta_{a}^{b} \delta_{j}^{i} \delta^{3}(x, y),
$$

and thus we must have

$$
I\left(\left(\hat{A}_{0}\right)_{a}^{i}\right)=i \hat{A}_{a}^{i}+g_{a}^{i}(\hat{\tilde{E}})
$$


with $g_{a}^{i}$ a yet undetermined function of the densitized triad. Recalling the definition of the triadic extrinsic curvature, and employing Eq. (50) and $\Gamma_{a}^{i}(-i \hat{\tilde{E}})=\Gamma_{a}^{i}(\hat{\tilde{E}})$, we obtain that

$$
I\left(\left(\hat{K}_{0}\right)_{a}^{i}\right)=i \hat{K}_{a}^{i}+f_{a}^{i}(\hat{\tilde{E}}),
$$

where $f_{a}^{i} \equiv i g_{a}^{i}-(1+i) \Gamma_{a}^{i}$. It can be seen then that $I\left(\hat{K}_{0}^{*}\right)=\left(I\left(\hat{K}_{0}\right)\right)^{*}$ provided that $\bar{f}_{a}^{i}(\hat{\tilde{E}})=-f_{a}^{i}(\hat{\tilde{E}})$.

Equations (53) and (56) fix the isomorphism between $*$-algebras up to a function $f_{a}^{i}$. As we have commented above, this isomorphism must also leave invariant the constraints of general relativity, that is, Eqs. (37), (39) and (42). Given the similarity of Eqs. (53) and (56) with the *-relations (31) and (34), it is not difficult to realize that one can parallel the discussion of the previous section about the equivalence of the gravitational constraints and their *-conjugate by simply regarding the isomorphism $I$ as a $*$-operation of the type (31) and (34) with $\Omega^{4}=i$, and conclude therefore that, for the isomorphism to respect the constraints, one must have $f_{a}^{i}=0$ and

$$
q^{2}(\hat{\tilde{E}}) R(\hat{\tilde{E}})=0
$$

this last equation arising from condition (48) evaluated at $\Omega^{4}=i$. So, except in some particular cases, i.e., in flat minisuperspace models, there exists no isomorphism between the $*$-algebras associated with reality conditions (10) and (48) such that it maps the Euclidean Hermitian triad $i\left(\hat{\tilde{E}}_{0}\right)_{i}^{a}$ into the Lorentzian triad $\hat{\tilde{E}}_{i}^{a}$ and preserves in addition the gravitational constraints. Then, the sets of reality conditions that correspond to Euclidean and Lorentzian gravity determine inequivalent quantum theories, and it seems impossible to regain Lorentzian physics from the quantum theory obtained by using the Euclidean reality conditions. 


\section{Conclusions}

We have shown that the set of reality conditions that are usually associated with Euclidean gravity, i.e., that the densitized triad and the Ashtekar connection be Hermitian, are inconsistent with the algebra of commutators in the Ashtekar formulation of general relativity. We have also argued that the reality conditions are invariant under a Wick rotation of the time, and thus the quantum theories that describe Lorentzian gravity and its Euclideanized version are in fact completely equivalent.

We have considered then a family of classical theories that are related to Lorentzian and Euclidean gravity in vacuum by a constant conformal transformation. If one requires that the classical real triad is represented by a Hermitian operator in these theories, the compatibility of the $*$-operation with the algebra of commutators and constraints restricts the admissible complex conformal factors to be either real or purely imaginary. For real conformal factors, one recovers the Lorentzian section of general relativity and its well-known set of reality conditions. In the case of a purely imaginary conformal factor, the classical theory can be identified as real Euclidean gravity, and the corresponding set of reality conditions guarantee that any real observable derived from the Euclidean 3-metric and extrinsic curvature is self-adjoint in the quantum theory. Therefore, these reality conditions can be interpreted as those associated with Euclidean general relativity. Explicitly, the Euclidean Ashtekar connection must be Hermitian, and the densitized triad has to be represented by an anti-Hermitian operator.

Finally, we have proved that the Lorentzian and the Euclidean sets of reality conditions lead to inequivalent quantum theories once one identifies the operators that describe the classical 3-metric in these two different quantizations. It thus seems impossible to extract Lorentzian physical predictions from the quantum theory 
determined by the Euclidean reality conditions, which would have been much simpler to impose when proceeding to the quantization of general relativity.

\section{Acknowledgements}

The author is greatly thankful to A. Ashtekar, F. Barbero and D. Marolf for helpful discussions. He wants to thank also the Center of Gravitational Physics and Geometry at the Pennsylvania State University for warm hospitality. This work

was supported by funds provided by the Spanish Ministry of Education and Science Grant No. EX92-06996911. 


\section{References}

[1] A. Ashtekar, Phys. Rev. Lett. 57, 2244 (1986).

[2] A. Ashtekar, Phys. Rev. D36, 1587 (1987).

[3] A. Ashtekar, Lectures on Non-Perturbative Canonical Gravity, eds. L. Z. Fang and R. Ruffini (World Scientific, Singapore, 1991).

[4] A. Ashtekar, in Gravitation and Quantizations, Les Houches Summer School Proceedings Vol. LVII, eds. B. Julia and J. Zinn-Justin (North-Holland, Amsterdam, 1993).

[5] T. Jacobson and L. Smolin, Nucl. Phys. B299, 295 (1988).

[6] C. Rovelli and L. Smolin, Phys. Rev. Lett. 61, 1155 (1988);Nucl. Phys. B331, 80 (1990).

[7] C. Rovelli, Class. Quantum Grav. 8, 1613 (1991).

[8] J. Zegaard, Nucl. Phys. B378, 288 (1992); B. Brügmann, R. Gambini and J. Pullin, Phys. Rev. Lett. 68, 431 (1992).

[9] P. A. M. Dirac, Lectures on Quantum Mechanics, Belfer Graduate School of Science Monograph Series No. 2 (Yeshiva University, New York, 1964).

[10] A. Rendall, Syracuse University preprint SU-GP-93/2-2 (1993).

[11] A. Ashtekar, J. D. Romano and R. S. Tate, Phys. Rev. D40, 2572 (1989).

[12] S. W. Hawking, in General Relativity. An Einstein Centenary Survey, eds. S. W. Hawking and W. Israel (Cambridge University Press, Cambridge, 1979).

[13] S. W. Hawking, Commun. Math. Phys. 87, 395 (1982).

[14] R. M. Wald, General Relativity (University of Chicago Press, Chicago, 1984). 
[15] S. W. Hawking, Nucl. Phys. B 239, 257 (1984).

[16] J. Glimm and A. Jaffe, Quantum Physics. A Functional Integral Point of View (Springer Verlag, New York, 1981).

[17] J. J. Halliwell, in Quantum Cosmology and Baby Universes, Proceedings of the Seventh Jerusalem Winter School, eds. S. Coleman, J. B. Hartle and T. Piran (World Scientific, Singapore, 1991).

[18] J. B. Hartle and S. W. Hawking, Phys. Rev. D28, 2960 (1983).

[19] H. Kodama, Phys. Rev. D42, 2548 (1990).

[20] G. A. Mena Marugán, Penn State University preprint CGPG-93/9-3 (1993). 